\title{
Extraction and Separation of Nonstationary Signals in Different Linear Mixed Models Based on Time-Frequency Analysis
}

\author{
Hui Zhang ${ }^{1}$ \\ ${ }^{1}$ Jiangsu Vocational College of Medicine, Yancheng, 224006, China
}

Keywords: Time-Frequency analysis; Linear mixed model; Non-Stationary signal

\begin{abstract}
Nonstationary signal analysis and processing are widely used in noise reduction, feature extraction, state recognition, fault diagnosis and other fields. The general methods include time domain analysis, frequency domain analysis and time-frequency combined analysis. Time frequency analysis is an ideal signal analysis method. In this paper, the definition and idea of time-frequency analysis are introduced. Short-time Fourier Transform (STFT) and wavelet transform (WT) linear hybrid models for time-frequency analysis are described. Finally, the future development of time-frequency analysis is introduced.
\end{abstract}

\section{Introduction}

Signal processing is one of the fastest developing subjects in information science. There are three basic assumptions in traditional statistical signal processing: linear, Gauss and stable. Modern signal processing takes nonlinear, non-Gauss and non-stationary signals as the object of analysis and processing. In modern signal processing, the development of non-stationary signal processing is particularly significant. According to the time characteristics of signal statistics, random signals can be divided into stationary random signals and non-stationary random signals. Nonstationary signal refers to the signal that its distribution parameter or distribution rule varies with time. Stationary and non-stationary signals are random. The most direct meaning of signal analysis is to find out the characteristics of different signals by analyzing or testing methods, so as to understand their characteristics and grasp their changing regularity. In short, the signals are identified objectively. Therefore, by means of signal analysis, complex signals can be decomposed into the sum of several simple signal components. Or a finite set is used to represent complex waveform signals. As a branch of modern signal processing, time-frequency analysis plays a very important role in signal processing. It combines time domain and frequency domain to represent signals in the form of joint time-frequency distribution. It overcomes the traditional single signal analysis fault in time domain or frequency domain, so that the signal characteristics can be clearly displayed on the time-frequency plane.

\section{Introduction to Time-Frequency Analysis}

\section{Definition of time frequency analysis}

Time-frequency analysis (JTFA), as a powerful tool for analyzing time-varying non-stationary signals, has become a hotspot of modern signal processing research. As a new signal processing method, it has attracted more and more attention in recent years. Time-frequency analysis provides joint distribution information in time and frequency domains, and clearly describes the relationship between signal frequency change and time.

\section{Basic idea of time-frequency analysis}

The basic idea of time-frequency analysis is to design a joint function of time and frequency, and use it to describe the energy density or intensity of a signal at different times and frequencies. The combined effect of time and frequency is simply called time frequency distribution. The time-frequency distribution is used to analyze the signal. It shows that the energy accumulates near the instantaneous frequency, and the time-frequency filtering and time-varying signal synthesis can be carried out. 


\section{Two Linear Mixed Models Based on Time-Frequency Analysis for Effective Feature Extraction}

\section{Short time Fourier transform}

Short time Fourier transform is one of the most important tools in time-frequency analysis. The difference between the Fourier transform and the Fourier transform is that the Fourier transform only provides the information of the frequency component, but not the time information. The short time Fu Liye transform clearly provides both. This time-frequency analysis method is conducive to signal analysis, and its frequency varies with time.

The basic idea of STFT is to localize the time domain. Based on Fourier transform, the time-varying signal $(t)$ is multiplied by the time window function $g(t)$. If the window function width is narrow enough, the signal can be regarded as stationary. The short time Fourier transform can be obtained by sliding the time window function on the time axis.

The definition is:

$$
\operatorname{STFT}_{s}(t, w)=\int s(\tau) g^{*}(\tau-t) \exp \{-j w \tau\} d \tau
$$

Among them, * represents complex conjugate and $\mathrm{g}(\tau)$ is a sliding window function. Hamming windows, Gauss windows and index windows are often used. From equation (3.1), it can be seen that the STFT of signal $\mathrm{s}(\tau)$ at time $\mathrm{t}$ is the Fourier transform of signal multiplied by a t-centered "analysis window" $g(\tau-\mathrm{t})$. Therefore, STFTs $(\mathrm{t}, \mathrm{w})$ can be understood as a signal $\mathrm{s}(\tau)$ near the analysis time $t$ Fourier transform, that is, local spectrum.

Thus the STFT transform of LFM signal is obtained.

$$
\operatorname{STFT}_{s}(t, w)=\int \operatorname{rect}\left(\frac{t}{T_{p}}\right) g^{*}(\tau-t) \exp \left[j\left(2 \pi f_{c} t+\frac{1}{2} \not t^{2}-w \tau\right)\right] d \tau
$$

STFT can also be implemented in frequency domain using filter banks.

$$
\operatorname{STFT}(t, w)=\frac{1}{2 \pi} e^{j w t} \int S(\Omega) G^{*}(\Omega-w) e^{j \Omega t} d \Omega
$$

In formula (3.3), $\mathrm{s}(\mathrm{t})$ and $\mathrm{g}(\mathrm{t})$ are Fu Liye transforms of $\mathrm{S}(\Omega)$ and $\mathrm{G}(\Omega)$ respectively.

When the window function satisfies the following conditions:

$$
\int g^{*}(t) f(t)=1
$$

$\mathrm{f}(\mathrm{t})$ is a composite window function, and it has to meet the requirement of formula (3.5) with the analysis window function $\mathrm{g}(\mathrm{t})$. Let $\mathrm{s}(\mathrm{n})$ be a discrete time signal. Consider the sampling of STFT at intervals $[\mathrm{mT}, \mathrm{nF}] . \mathrm{T}>0$ and $\mathrm{F}>0$ are sampling periods of time and frequency respectively. $\mathrm{m}$ and $\mathrm{n}$ are integers. The discrete STFT analysis formula can be obtained.

$$
\operatorname{STFT}_{s}[m T, n F]=\sum_{k} s[k] g^{*}[k T-m T] e^{-j 2 \pi(n F) k}
$$

The corresponding discrete STFT reconstruction formula is:

$$
s[k]=\sum_{m} \sum_{n} S T F T s[m T, n F] g[k T-m T] e^{j 2 \pi(n F) k}
$$

Because STFT analysis is based on static signals, the window width is very narrow in both time domain and frequency domain. Otherwise, it is difficult to get the approximate result of the signal spectrum in a certain time or in the waveform corresponding to a certain frequency component. The narrower the time window is, the higher the temporal resolution. However, due to its inverse ratio, the frequency window will narrow. Similarly, in the frequency domain, the narrower the frequency window is, the higher the frequency resolution, but the time resolution will decrease. Because the uncertainty principle does not include the existence of window functions with short-time and narrow-bandwidth, high temporal and frequency resolution cannot be obtained simultaneously, and it should be destroyed as needed.

\section{Wavelet transform}

The wavelet is completely defined by a zoom filter $\mathrm{g}$, which has a low pass finite impulse response (FIR) of $2 \mathrm{~N}$ and 1 in length. In the case of biorthogonal wavelets, the filtering of 
decomposition and reconstruction is defined respectively. The analysis of the high pass filter is calculated as low pass QMF, while the reconstruction filter is the time reversal of decomposition, such as Daubechies and Symlet wavelets. The wavelets of time domain wavelet function ( $\mathrm{t}$ ) and zoom function $\Phi(\mathrm{t})$ are defined. The wavelet function is actually a bandpass filter, and each successive scaling reduces the bandwidth by half. This leads to an infinite number of problems covering the entire spectrum. The scaling function filters out the lowest level of transformation and ensures that the entire spectrum is covered. For compactly supported wavelets, $\Phi(t)$ can be regarded as finite length and equivalent to the scaling filter $\mathrm{g}$, such as, Meyer wavelet. Wavelets are represented only in time domain as wavelet functions $\psi(t)$.

\section{Conclusion}

With the rapid development of information science and computer technology, signal processing and application have made great progress. As one of the most important processing methods in modern signal processing, time-frequency analysis has been applied in more and more fields. More and more researchers will explore it further. From its development point of view, with the passage of time, time-frequency analysis method, like other scientific methods, will become more and more perfect.

\section{Reference}

[1] Stankovic L, Orovic I, Stankovic S, et al. Compressive Sensing Based Separation of Nonstationary and Stationary Signals Overlapping in Time-Frequency[J]. IEEE Transactions on Signal Processing, 2013, 61(18):4562-4572.

[2] Li Z, Yan X, Tian Z, et al. Blind vibration component separation and nonlinear feature extraction applied to the nonstationary vibration signals for the gearbox multi-fault diagnosis[J]. Measurement Journal of the International Measurement Confederation, 2013, 46(1):259-271.

[3] Zhang H, Bi G, Razul S G, et al. Robust time-varying filtering and separation of some nonstationary signals in low SNR environments[J]. Signal Processing, 2015, 106(C):141-158.

[4] Nie W, Li Y, Liu D. A blind source separation algorithm of non-stationary signals based on local polynomial Fourier transform[C]// Progress in Electromagnetic Research Symposium. IEEE, 2016:4996-5000.

[5] Saulig N, Milanovic Z, Mauša G. Performance Comparison of Blind Source Separation Algorithms for Nonstationary Signals[C]// International Conference on Innovative Technologies. 2015.

[6] Liang L P, Xu K J, Xu W. SWT based separation method for periodic signal with non-stationary noise and its application in EMF[J]. Flow Measurement \& Instrumentation, 2015, 42:78-88. 